\title{
On the light echo in V838 Mon
}

\author{
R. Tylenda* \\ N. Copernicus Astronomical Center, Department for Astrophysics, Rabiańska 8, 87-100 Toruń, Poland \\ Received 26 June 2003 / Accepted 30 September 2003 \begin{abstract}
study the available images of the light echo in V838 Mon obtained with HST. An investigation of the observed expansion of the light echo leads us to conclude that the distance to V838 Mon is $\gtrsim 5 \mathrm{kpc}$. From an analysis of the inner bright echo rim asymmetric dust-free region which we interpret as produced by a fast wind from the V838 Mon system. The asymmetry would
\end{abstract} \\ Abstract. We present a theoretical outline of the light echo phenomenon and results of simple numerical simulations and we \\ we estimate the distance to be $8.0 \pm 2.0 \mathrm{kpc}$. We also investigate the structure of the dust distribution in the vicinity of the \\ object. We find no obvious signs of spherical symmetry in the resultant distribution. Near the central object there is a strongly \\ imply that V838 Mon is moving relative to the dusty medium. From these results we conclude that the dust illuminated by the \\ light echo is of interstellar origin rather than produced by mass loss from V838 Mon in the past.
}

Key words. stars: individual: V838 Mon - stars: distances - stars: circumstellar matter - ISM: reflection nebulae

\section{Introduction}

V838 Mon was discovered in eruption in the beginning of January 2002 (Brown 2002). The main outburst, however, started at the beginning of February 2002 and, in the optical, lasted for about two months (e.g. Munari et al. 2002a). The nature of the eruption is enigmatic (e.g. Munari et al. 2002a; Kimeswenger et al. 2002). It cannot be accounted for by known thermonuclear events (classical nova, late He-shell flash) and therefore new, as yet unexplored, mechanisms have been searched for (Soker \& Tylenda 2003; Retter \& Marom 2003).

V838 Mon has recently received significant publicity, even in non-scientific media, due to its light echo. This event was discovered shortly after the main eruption in February 2002 (Henden et al. 2002). However, the most spectacular images of the V838 Mon light echo were provided later by HST (Bond et al. 2003).

The light echo is a rare event and can be observed when a light outburst illuminates circumstellar or interstellar dust. So far it has primarily been observed for extragalactic supernovae (e.g. Crotts 1988; Xu et al. 1995; Schmidt et al. 1994; Cappellaro et al. 2001; Sugerman \& Crotts 2002). In our Galaxy this phenomenon has been observed in Nova Persei 1901 (e.g. Couderc 1939).

Analyses of light echoes can be used while investigating supernova light curves (e.g. Chevalier 1986). Observed evolution of the light echo arround SN 1987A in LMC has enabled one to study the dust distribution in front of the supernova up to a distance of $\sim 1 \mathrm{kpc}$ from the supernova (see e.g. Xu et al. 1995).

\footnotetext{
* e-mail: tylenda@ncac.torun.pl
}

Supernova light echoes can also be used to measure distances to galaxies (Sparks 1994).

Munari et al. (2002a) and Kimeswenger et al. (2002) have attempted to use the observed expansion of the light echo arround V838 Mon to measure the distance. Their calculations were based on a naive interpretation of the light echo expansion (i.e. that it expands at the velocity of light) and the derived values of $0.6-0.8 \mathrm{kpc}$ are in fact significant underestimates of the distance. A more realistic analysis in Bond et al. (2003) gave a lower limit to the distance of $\sim 6 \mathrm{kpc}$.

In this paper we present an analysis of the available data on the light echo in V838 Mon. After presenting theoretical considerations and results of simple simulations of light echoes (Sect. 2) we attempt to use the observational data to constrain the distance to V838 Mon (Sects. 3 and 4.2) and to study the dust distribution near the object (Sect. 4). The results are summarized and discussed in Sect. 5.

\section{Basic considerations and numerical simulations}

Couderc (1939) was probably the first to give a correct explanation for the light echo arcs observed arround Nova Persei 1901. More recently, theoretical considerations on the light echo can be found in papers devoted to supernova light echoes (e.g. Chevalier 1986; Sparks 1994). The subject is however relatively unknown in the field of stellar astrophysics. This is probably the reason why in some papers oversimplified or even incorrect interpretations of the V838 Mon light echo can be found. Therefore we summarize basic formulae describing the structure and evolution of the light echo. 


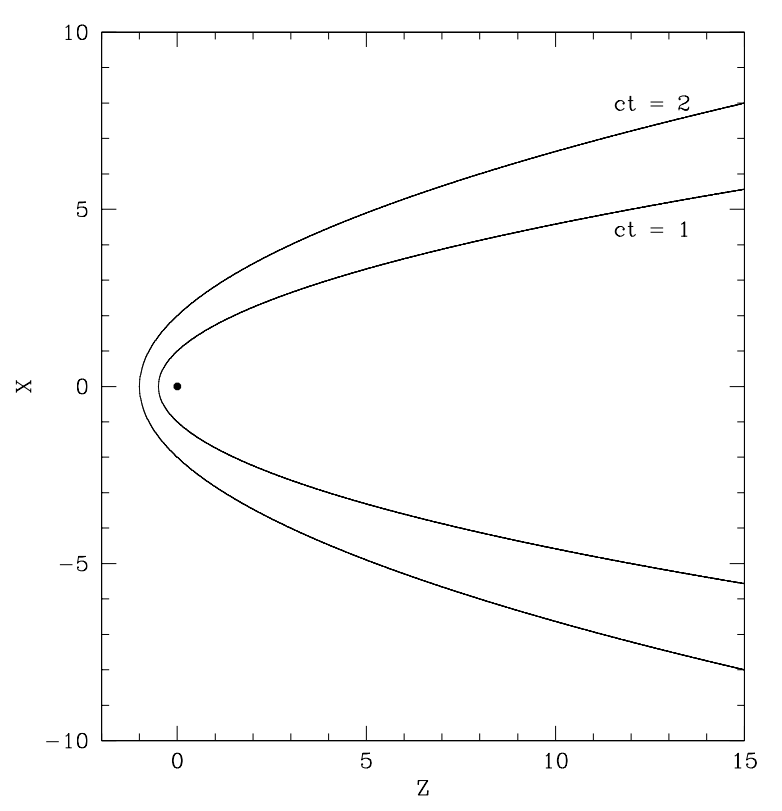

Fig. 1. The light echo paraboloid (Eq. (3)) shown in the $x z$ plane for $c t=1$ and $c t=2$. The axes are in units of $c t$. The dot marks the source position at $x=0, z=0$. The observer is at $x=0, z=\infty$.

We define a rectangular $(x, y, z)$ coordinate system with its origin being in the light source. The $z$ axis is along the line of sight toward the observer. The $(x, y)$ plane is perpendicular to the $z$ axis, i.e. is a tangent to the sky sphere in the source. Let the source emits short light flash at time $t=0$. We assume the so-called single scattering approximation, i.e. that photons are scattered only once in the dusty medium surrounding the source. Suppose that the scattered echo is seen by the observer at a time $t$. Then the illuminated dust lies on a surface defined by

$r+l=d+c t$

where $c$ is the velocity of light, $d$ is the distance between the source and the observer, $r=\sqrt{x^{2}+y^{2}+z^{2}}$ is the distance of the scattering dust from the source, and $l=\sqrt{x^{2}+y^{2}+(d-z)^{2}}$ is the distance of the dust from the observer. Equation (1) is an ellipsoid having foci in the source and the observer.

The considerations can be simplified when $x$ and $y$ are much smaller than $d$, i.e. when the observed angular dimensions of the echo are small. Setting $l=d-z$ Eq. (1) becomes

$r=z+c t$

or equivalently

$x^{2}+y^{2}=(c t)^{2}+2 z c t$.

This is a paraboloid symmetric around the $z$ axis (line of sight) and having its focus in the source. In the case of V838 Mon the echo has dimensions of order of $1^{\prime}$ so in this case the relative accuracy of Eqs. (2) and (3) is better than $10^{-3}$.

Figure 1 shows Eq. (3) in the $x z$ plane for $c t=1$ and $c t=2$ (axes in the figure are in units of $c t$ ). Several important conclusions can be drawn from Fig. 1. If the source (at $x=0, z=0$ ) produced a short flash of light at $t=0$ then at $t=1 / c$ and $t=2 / c$ the observer would see dust illuminated on paraboloids $c t=1$ and $c t=2$, respectively. However, if the flash started at $t=0$ and lasted up to $t=1 / c$ then at $t=1 / c$ all dust within the paraboloid $c t=1$ would be illuminated for the observer. At $t=2 / c$ the illuminated dust would be between the paraboloids $c t=1$ and $c t=2$. Thus contrary to naive considerations the echo does not expand spherically starting from the source. It starts from the line of sight, i.e. $x=0, z \geq 0$, and most effectively penetrates regions situated in front of the source. Behind the source the penetration is slowest.

The observed echo is in the $(x, y)$ plane and its structure and evolution depends on the dust distribution around the source. An important conclusion drawn from Fig. 1 is that if the observed echo has a well defined outer edge (as observed in the case of V838 Mon) the dusty medium around the source has to have a rather well defined outer boundary in front of the source.

In order to illustrate dependence of the light echo structure and evolution on the dust distribution let us consider two simple dust geometries, i.e. a plane-parallel slab and a spherical shell centred on the source.

\subsection{Plane-parallel dust slab}

Let us assume that a thin plane-parallel slab of dust intersects the line of sight at $z_{0}$ and that the normal to the slab is inclined to the $z$ axis at an angle $\alpha$. Let us also assume, for simplicity, that the normal lies in the $(x, z)$ plane. The slab is then described by

$z=z_{0}-a x$

where $a=\tan \alpha$. Equation (4), when used to eliminate $z$ from Eq. (3), gives the echo shape as follows

$x^{2}+y^{2}=(c t)^{2}+2 z_{0} c t-2 a x c t$.

Thus the echo is in the shape of a ring whose radius, $\xi$, is given by

$\xi=\sqrt{\left(1+a^{2}\right)(c t)^{2}+2 z_{0} c t}$.

The ring is centred at

$x_{\mathrm{c}}=-a c t, y_{\mathrm{c}}=0$.

The velocity of expansion of the echo ring, $v$, is

$v \equiv \frac{\mathrm{d} \xi}{\mathrm{d} t}=c \frac{\left(1+a^{2}\right) c t+z_{0}}{\xi}=c \frac{\left(1+a^{2}\right) c t+z_{0}}{\sqrt{\left(1+a^{2}\right)(c t)^{2}+2 z_{0} c t}}$.

An analysis of Eqs. (6)-(8) leads to the following conclusions. The echo ring is centred on the source only if the dust slab is perpendicular to the line of sight. In almost all the cases the echo expansion is superluminal. Only if $z_{0}=0$ and $a=0$ the echo expands at $c$. For $z_{0}>0$ (the slab in front of the source) the echo appears at the same time as the source flash, i.e. at $t=0$. It starts expanding from the source position, i.e. from $x_{\mathrm{c}}=0, y_{\mathrm{c}}=0$. For $z_{0}<0$ (the slab behind the source) the echo appears with a time delay relative to the source flash, i.e. it starts at $t=\left(-2 z_{0}\right) /\left(\left(1+a^{2}\right) c\right)$. In this case it begins expanding from the position $x_{\mathrm{c}}=\left(2 z_{0} a\right) /\left(1+a^{2}\right), y_{\mathrm{c}}=0$. In both cases the 
expansion velocity is $\infty$ at the moment of the echo appearance, i.e. at $t=0$ if $z_{0}>0$ and at $t=\left(-2 z_{0}\right) /\left(\left(1+a^{2}\right) c\right)$ if $z_{0}<0$. It decreases asymptotically to $c \sqrt{1+a^{2}}$ when $\left(1+a^{2}\right) c t \gg$ $\left|z_{0}\right|$. For slabs non-perpendicular to the line of sight, i.e. when $a \neq 0$, the echo centre moves away from the source at a constant velocity of $|a| c$. The direction of this movement is opposite to the direction of the slab normal projected on the sky surface.

\subsection{Spherical dust shell}

Let us assume that a thin spherically symmetric shell of dust having a radius, $r_{0}$, is centred on the source, so it is described by

$r_{0}^{2}=x^{2}+y^{2}+z^{2}$.

This when used to eliminate $z$ from Eq. (3) gives

$x^{2}+y^{2}=2 r_{0} c t-(c t)^{2}$.

Thus the echo is in the shape of a ring with the radius, $\xi$, given by

$\xi=\sqrt{2 r_{0} c t-(c t)^{2}}$

and centred on the source $\left(x_{\mathrm{c}}=0, y_{\mathrm{c}}=0\right)$. The echo expansion velocity, $v$, is

$v \equiv \frac{\mathrm{d} \xi}{\mathrm{d} t}=c \frac{r_{0}-c t}{\xi}=c \frac{r_{0}-c t}{\sqrt{2 r_{0} c t-(c t)^{2}}}$.

As can be seen from Eq. (11), the echo radius starts from zero at $t=0$, reaches a maximum equal to $r_{0}$ at $t=r_{0} / c$, and collapses to zero at $t=2 r_{0} / c$. Note that in a coordinate system $\left(c t-r_{0}, \xi\right)$ Eq. (11) describes a circle centred on the origin and having the radius equal to $r_{0}$. The expansion velocity of the echo ring starts from $\infty$ at $t=0$, decreases and reaches zero at $t=r_{0} / c$, and continues decreasing to $-\infty$ at $t=2 r_{0} / c$.

\subsection{Numerical simulations}

To illustrate the evolution of the echo in different geometries of the dust distribution we have performed simple numerical simulations based on the theoretical considerations outlined above. The structure of the light echo has been determined in the paraboloid approximation, i.e. using Eq. (3), and adopting the single scattering approximation.

If the central source radiates at a luminosity, $L_{v}$, the intensity, $I_{v}$, of the radiation scattered at a point $(x, y, z)$ can be calculated from

$I_{v}=\frac{\kappa_{v} L_{v}}{4 \pi r^{2}}$,

where $\kappa_{v}$ is the scattering coefficient which is usually written in the form (e.g. Chevalier 1986)

$\kappa_{v}=n_{\mathrm{s}} \sigma_{\mathrm{s}} Q_{v} F_{v}(\phi)$

where $n_{\mathrm{s}}$ is the number density of scattering particles, $\sigma_{\mathrm{s}}$ is the particle cross section, $Q_{v}$ is the scattering efficiency (albedo), $F_{v}(\phi)$ is the scattering phase function for the scattering angle $\phi$ defined as $\cos (\phi)=z / r$. Integrating Eq. (13) along the $z$ axis over the dusty medium gives the echo surface brightness, in units of flux per unit solid angle, at any given time.

The simulations have been done for two simple geometries of the dust distribution, i.e. plane-parallel slabs and concentring shells. In all cases it has been assumed that dust is uniformly distributed, i.e. $\kappa_{v}=$ const., in slabs or shells and that scattering is isotropic, i.e. $F(\phi)=1 / 4 \pi$. Note that the simulations adopt very simple scenarios and have not been meant to mimic the situation observed in V838 Mon, although parameters like times and distances are of the order of those seen or expected in this particular case.

Figure 2 presents the simulated evolution of the echo structure from a model in which dust is distributed in four planeparallel slabs perpendicular to the $z$ axis. The $z_{0}$ positions of the slabs are $-0.10,0.00,0.30$, and $1.00 \mathrm{pc}$. The slab thickness, $\Delta z_{0}$, is the same in all cases and is equal to 0.02 pc. $L_{v}$ has been adopted to be constant during the source flash which lasted $\Delta t=50$ days (otherwise $L_{v}=0$ ). Figure 2 shows the echo structure as observed from a distance of $5 \mathrm{kpc}$ at 50,150 , $250,300,400$, and 500 days after the beginning of the flash. The grey scale is logarithmic and it has been normalized to the maximum surface brightness in the images. Between the brightest centre of the first image and the faintest outer ring in the last image there is a span of 4 orders of magnitude in the surface brightness.

The first image in Fig. 2 shows the echo just at the end of the source flash. According to the discussion of Fig. 1 all dust being within the paraboloid defined by the time elapsed since the beginning of the flash, is illuminated. Thus the echo is composed of three filled circles produced by three slabs having $z_{0} \geq 0.0$ (the fourth slab at $z_{0}=-0.1 \mathrm{pc}$ is not seen as it has not yet been reached by the light paraboloid). Later on the illuminated dust is situated between two paraboloids, i.e. the first one corresponding to the begining of the flash and the second one defined by the epoch of the flash end. Thus in the second image, taken 100 days after the end of the flash, the echo consists of three rings. The slab at $z_{0}=-0.10 \mathrm{pc}$ is reached by the first paraboloid at $t \simeq 210$ days, whereas the second paraboloid starts leaving it at $t \simeq 310$ days. Therefore this slab is seen in the form of a central disc in the third and fourth image. Later on it produces the innermost ring in the image.

As can be seen from Fig. 2 the surface brightness of the rings decreases with time but the rate of fading depends on $z_{0}$. The reason for the fading is, of course, the increasing geometric dilution. For a thin slab the surface brightness of the scattered radiation, $\Sigma_{v}$, is just $I_{v} \Delta z_{0}$. Thus, in general case, using Eqs. (13), (2) and (4) one finds

$\Sigma_{v} \propto \frac{\Delta z_{0}}{r^{2}}=\frac{\Delta z_{0}}{\left(z_{0}-a x+c t\right)^{2}}$.

Consequently, in our simulations the surface brightness in the ring corresponding to $z_{0}=0.0$ decreases by factor $\sim 500$ between the first image and the last one in Fig. 2, while the same drop in the case of the outermost ring $\left(z_{0}=1.00 \mathrm{pc}\right)$ is only factor $\sim 2$. With time the echo rings would continue expanding with the velocity aproaching $c$ according to Eq. (8) and the surface brightness decreasing as described by Eq. (15). 

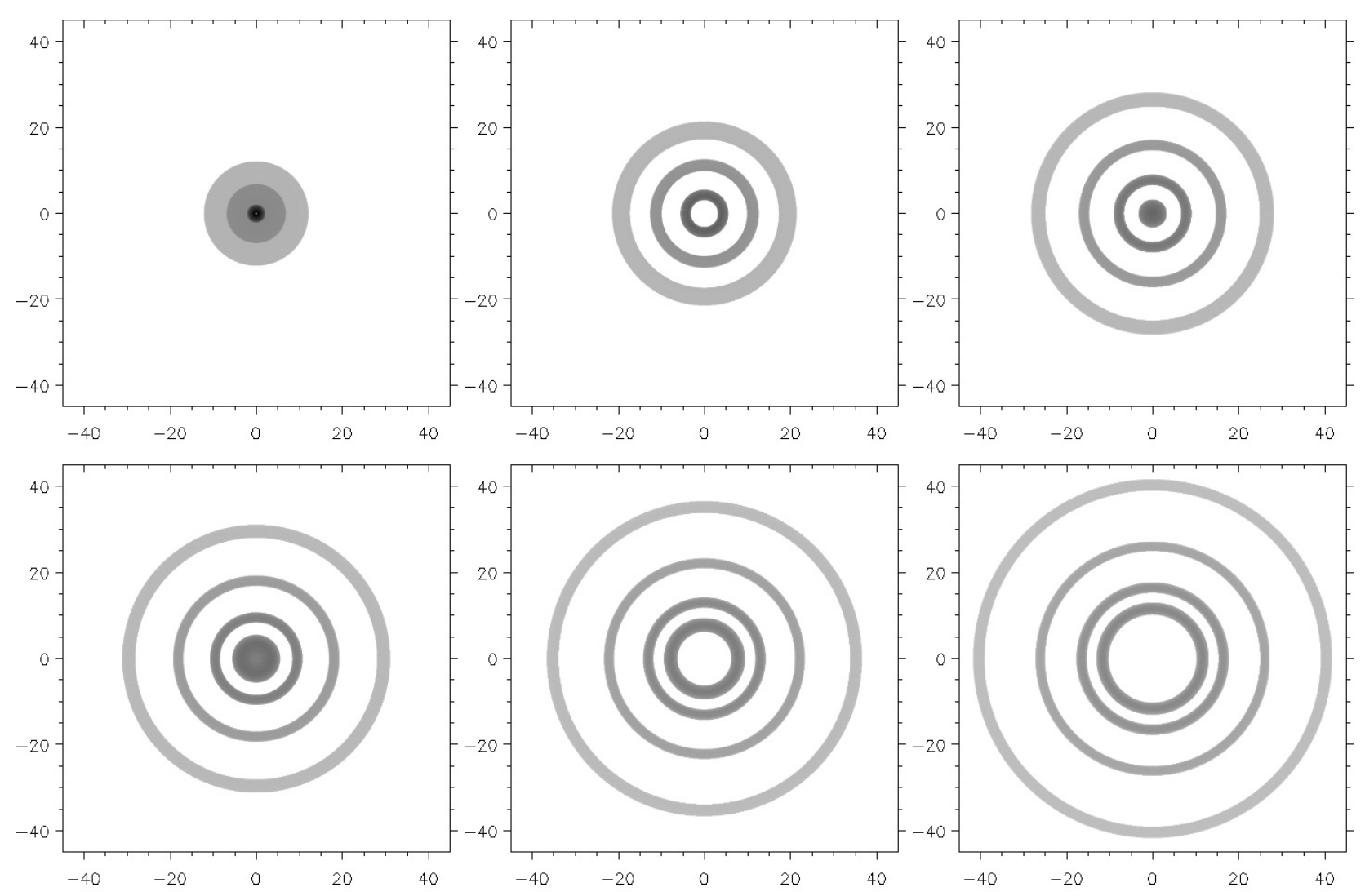

Fig. 2. The evolution of the echo structure produced by four dust slabs perpendicular to the line of sight at the $z$ positions of $-0.10,0.00$, 0.30 , and $1.00 \mathrm{pc}$. The source (not shown in the figure but lying in the centre of each image) has experienced a 50 days long flash of constant luminosity. All the slabs have the same thickness of $0.02 \mathrm{pc}$ and the same value (constant within the slabs) of the scattering coefficient $\sigma_{v}$. The object is assumed to be observed from a distance of $5 \mathrm{kpc}$. The images are ordered from upper-left to lower-right and correspond to the time epochs of 50, 150, 250, 300, 400, and 500 days after the beginning of the source flash. The axes of the images are in arcsec. The grey scale is logarithmic and is normalized to the brightest region in the figure. The brightest centre of the first image and the faintest outer ring in the last images span $\sim 4$ orders of magnitude in the surface brightness.

Figure 3 shows the evolution of the echo structure produced by three spherically symmetric dust shells centred on the source. The radii of the shells, $r_{0}$, are $0.10,0.30$, and $1.00 \mathrm{pc}$. All shells have the same thickness $\Delta r_{0}=0.02 \mathrm{pc}$. As in the previous case the source flash has lasted $\Delta t=50$ days and the object is observed from a distance of $5 \mathrm{kpc}$. The consecutive images correspond to the epochs of 50,150,250,400,600, and 750 days after the beginning of the source flash.

As in Fig. 2 the first image in Fig. 3 corresponds to the end of the source flash and all the dust within the light paraboloid produced by the beginning of the flash is illuminated. The echo is thus composed of three filled circles produced by illuminated sections of the corresponding shells. The echo components initially expand superluminally. However, as discussed in Sect. 2.2, the expansion rate of an echo produced by a shell slows down to 0 when reaching the maximum radius equal to the value of $r_{0}$. For the shells in our simulations, i.e. having $r_{0}=0.1,0.3$, and $1.0 \mathrm{pc}$, it happens at $t \simeq 130,370$, and 1200 days, respectively. After this, the echo ring starts shrinking and finally disappears as a fast collapsing disc. In our simulations the echoes due to the shells having $r_{0}=0.1,0.3$, and $1.0 \mathrm{pc}$ disappear at $t=310,790$, and 2450 days, respectively. The echo from the innermost shell is close to its maximum radius in the second image of Fig. 3 whereas in the third image it is collapsing. The echo from the shell having $r_{0}=0.3 \mathrm{pc}$ is near its maximum radius in the fourth image whereas its collapse is observed in the last two images.

The echo rings in our simulations reach maximum surface brightness at the maximum radius, i.e. at $t \simeq r_{0} / c$. At this time $\Sigma_{v}$ is about twice as high as in the begining of the echo evolution, i.e. at $t<\Delta t$. In general case, however, the evolution of $\Sigma_{v}$ depends on the ratio of $c \Delta t / \Delta r_{0}$. To show this let us consider a short flash, i.e. $\Delta t \ll r_{0} / c$, illuminating a thin shell, i.e. $\Delta r_{0} \ll r_{0}$. It is quite straightforward to show from simple geometric considerations and Eq. (2) that the light echo illuminates a constant part of the shell equal to $2 \pi r_{0} c \Delta t$. Thus the total flux scattered by the shell is also constant with time and proportional to $c \Delta t \Delta r_{0} / r_{0}$. From Eq. (11) one can calculate the echo radii corresponding to possible combinations of $t$ and $t-\Delta t$ with $r_{0} \pm \Delta r_{0} / 2$. Taking extreme radii as the boundaries of the echo ring and retaining only linear terms of $c \Delta t$ and $\Delta r_{0}$ one gets that the surface of the echo ring is equal to $2 \pi c \Delta t\left(\left|r_{0}-c t\right|+t \Delta r_{0} / \Delta t\right)$. Consequently the surface brightness of the echo varies as

$\Sigma_{v} \propto\left(\frac{r_{0}}{\Delta r_{0}}\left|r_{0}-c t\right|+\frac{r_{0}}{\Delta t} t\right)^{-1}$ 

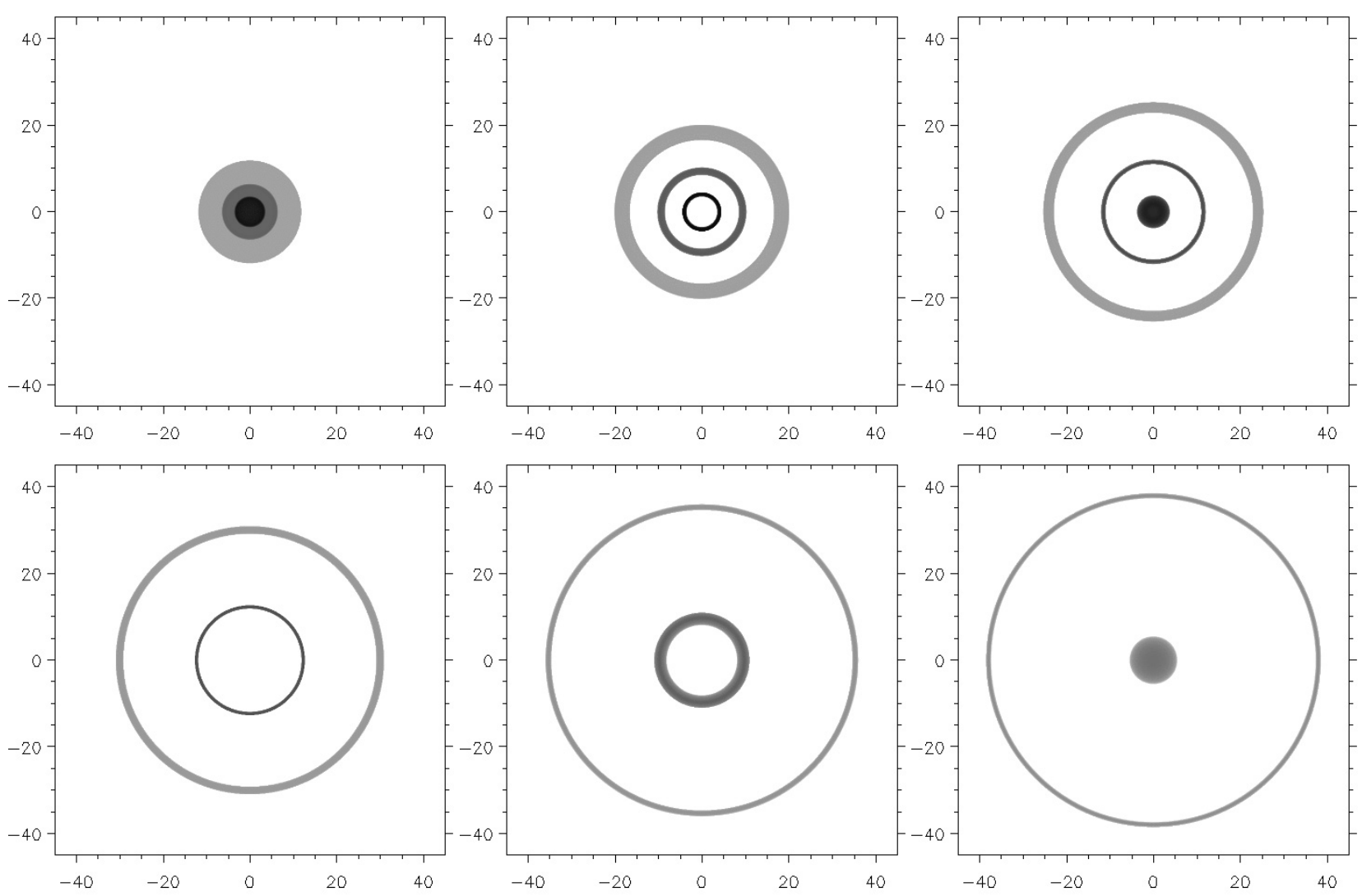

Fig. 3. The same as Fig. 2 but for three spherical shell of dust centred on the source. The shells have the same thickness of 0.02 pc and radii of $0.10,0.30$, and $1.00 \mathrm{pc}$. The images shows the echo as observed 50, 150, 250, 400, 600, and 750 days after the beginning of the source flash. The brightest and the faintest regions in the figure span $\sim 2.5$ orders of magnitude in the surface brightness.

As can be seen from Eq. (16), in the begining of the echo evolution, i.e. at $t=0, \Sigma_{v} \propto \Delta r_{0} / r_{0}^{2}$. Near the maximum echo radius, i.e. at $t=r_{0} / c, \Sigma_{\nu} \propto\left(\Delta r_{0} / r_{0}^{2}\right)\left(c \Delta t / \Delta r_{0}\right)$. At the end, i.e. at $t=2 r_{0} / c, \Sigma_{v} \propto\left(\Delta r_{0} / r_{0}^{2}\right) /\left(1+2 \Delta r_{0} / c \Delta t\right)$. Between these extreme time moments the surface brightness evolves monotonically. For $\Delta r_{0}<c \Delta t$ the maximum surface brightness occurs at the maximum radius, i.e. at $t=r_{0} / c$. This is the case of our simulations where $\Delta r_{0} \simeq 0.5 c \Delta t$. If, however, $\Delta r_{0}>c \Delta t$ the maximum surface brightness takes place at $t=0$. The surface brightness is lowest at the end of the echo, i.e. at $t=2 r_{0} / c$.

\subsection{Discussion}

The above considerations, although done for simplified cases, demonstrate that long term observations of the evolution of the echo structure can provide information on the dust distribution and thus, indirectly, also on the origin of the dust producing the echo. If dust results from a past mass loss from the central object it would tend to show certain symmetries in its distribution around the source. Usually mass loss is not continuous at a constant rate but it takes place in the form of more or less periodic fluctuations of the mass loss rate, e.g. during the AGB phase, or discrete events of strong mass loss separated by longer periods of quiescence, e.g. in nova-like outbursts. Then the dust distribution would show a succession of shell-like structures or be in the form of a more or less defined envelope. The evolution of the light echo in such circumstances would follow the general patterns discussed in Sect. 2.2 and Fig. 3. The initially fast expansion, slowing down when reaching maximum sizes corresponding to outer edges of the dust shells or envelope, would be followed by an accelerated collapse.

If, however, dust is not related to the source and is rather of interstellar nature, no obvious symmetry with respect to the light source is expected. Instead dust would rather be confined in extended sheets, zones or filaments and our considerations of plane-parallel slab geometries in Sect. 2.1 and Fig. 2 would be more appropriate. The echo would expand more and more with slowly decreasing brightness as more and more distant regions are illuminated.

Note that for studying the general character of the dust distribution around the source the echo has to be observed for a long enough time. The initial evolution of the echo produced by spherical dust shells is similar to that due to slabs. The light paraboloid is close to the line of sight so the echo does not feel differences in the general shape of the dust distribution. This can be seen from Eqs. (6) and (11) which reduce to the same form if $c t \ll z_{0}$ and $c t \ll r_{0}$, respectively. Therefore the first image in Fig. 3 is not much different from that in Fig. 2 (the reason why the innermost (brightest) disc in Fig. 2 is significantly smaller than the corresponding one in Fig. 3 is that in the former case it is produced by the slab at $z_{0}=0.0$ whereas in the latter case the shell has $r_{0}=0.1 \mathrm{pc}$ ). Later on, when $c t$ 
is not any more negligible with respect to $z_{0}$ and $r_{0}$, the two geometries can be better disentangled from the echo evolution.

\section{Distance to V838 Mon constrained from the echo expansion}

An analysis of the light echo evolution can be used to estimate the distance to the object. An attempt to derive the distance to V838 Mon has been done by Bond et al. (2003), who measured expansion rates in ring-like structures in the echo from two consecutive images and found that the distance is greater than $2 \mathrm{kpc}$. Note, however, that this estimate assumed that the ring-like structures were produced by concentric shells which is not justified, as discussed below. The same authors have also applied the observed polarization structure of the echo, as proposed by Sparks (1994), which led them to conclude that the lower limit to the distance is $\sim 6 \mathrm{kpc}$.

We have attempted to put constraints on the distance to V838 Mon by measuring the outer echo edge expansion. As it is clear from the considerations in Sect. 2 the outer edge of the echo is produced by the beginning of the light flash reflected at the outermost edge of the dust distribution in front of the source.

For the plane slab geometry Eq. (6) when combined with Eq. (7) can be rewritten as

$\theta=\frac{\xi}{d}=\sqrt{\left(\frac{2 z_{0}}{c t}+1\right)\left(\frac{c t}{d}\right)^{2}+\theta_{\mathrm{c}}^{2}}$

where $d$ is the distance to the object, $\theta$ is the angular radius of the echo, and $\theta_{\mathrm{c}}=x_{\mathrm{c}} / d$ is the angular distance of the echo centre from the source. At early epochs, i.e. when $\left(2 z_{0}\right) /(c t) \gg$ 1 , Eq. (17) allows to determine only the value of $z_{0} / d^{2}$. Thus unless $z_{0}$ is known the distance cannot be determined. However, in later epochs, when $\left(2 z_{0}\right) /(c t) \ll 1$, Eq. (17) can be used to derive $d$ from the observed values of $\theta$ and $\theta_{\mathrm{c}}$.

In the case of the spherical symmetry Eq. (11) can be rewritten as

$\theta=\sqrt{\left(\frac{2 r_{0}}{c t}-1\right)\left(\frac{c t}{d}\right)^{2}}$.

Thus, not only in early phases (fast initial expansion) but also in late phases (final collapse) the distance cannot be determined unless the value of $r_{0}$ is known. Only near the maximum size of the echo, i.e. when $r_{0} \approx c t$, Eq. (18) can give a direct estimate of the distance.

In a real case, such as the one of V838 Mon, we do not know a priori what is the distance of the outer edge of dust from the source nor what is the geometry of this edge. The distance of the scattering dust could, in principle, be constrained from an analysis of the echo surface brightness as the amount of the radiation scattered depends on the distance squared (Eq. (13)). This, however, requires calibrated data which are not available to the author. Thus the only way is to look at the observed evolution of the echo radius and try to fit it with Eq. (17) or Eq. (18).

For this purpose we have measured the positions of the outer edge of the light echo of V838 Mon in
Table 1. Results of fitting a circle to the outer edge of the light echo of V838 Mon. Time of observations, $t_{\mathrm{obs}}$, is in days since January 1, 2002. Results are in arcsec.

\begin{tabular}{cccc}
\hline \hline$t_{\text {obs }}$ (days) & $\theta$ & $x$ (centre) & $y$ (centre) \\
\hline 120.0 & $18.55 \pm 0.76$ & $-0.84 \pm 1.10$ & $0.46 \pm 1.06$ \\
140.0 & $20.89 \pm 0.71$ & $-1.12 \pm 1.02$ & $0.51 \pm 0.98$ \\
245.0 & $30.32 \pm 0.92$ & $-2.27 \pm 1.34$ & $0.37 \pm 1.27$ \\
301.0 & $33.68 \pm 0.95$ & $-2.36 \pm 1.38$ & $1.11 \pm 1.31$ \\
351.0 & $36.66 \pm 1.01$ & $-2.61 \pm 1.46$ & $1.58 \pm 1.37$ \\
\hline
\end{tabular}

five images obtained by H.E. Bond and available on the HST web site (http://hubblesite.org/newscenter/ archive/2003/10/, see also Bond et al. 2003). They have been taken on April 30, May 20, September 2, October 28, and December 17, 2002. In each image the positions of typically $60-70$ points at the outer edge of the echo (more or less equally spaced in the azimuthal angle) have been measured. In most cases the outer echo edge is quite well defined and can be rather easily measured. However, in some instances, mostly in south-eastern directions, the edge is broken or composed of incomplete arcs. This introduces certain ambiguity in measurements and uncertainties in the results. Note that all the measurements (also those reported in the next section) have been done on negatives of the published images as the emission edges and other details in the light echo are then more easily seen.

The positions have been determined in the coordinate system centred on V838 Mon with the $x$ and $y$ axes pointing to west and north, respectively. Then a circle has been fitted to the measured positions in each image using the $\chi^{2}$ minimum method. The results are presented in Table 1. First column shows the time of observations, $t_{\mathrm{obs}}$, given in days since January 1,2002 . The radius of the echo, $\theta$, and its uncertainty are given in the next column. The last two columns show the position of the centre of the fitted circle relative to the central star. All the results are in arcsec.

In order to make a quantitative analysis of the results in Table 1, it is necessary to determine the time moment of the zero age of the echo $t_{0}$. The echo has been discovered in midFebruary 2002 (Henden et al. 2002) and it has been suggested that it results from the main outburst, which started at the beginning of February 2002 (Munari et al. 2002a; Bond et al. 2003). This is supported by the fact that in the HST images the outer echo rim is blue and it was at the beginning of the main outburst when the star was bluest (see e.g. Bond et al. 2003). The main outburst started on February 1 and the maximum was reached on February 5-6 (Munari et al. 2002a; Bond et al. 2003). Thus we adopt February 3 as the date of the zero age of the echo, i.e. $t_{0}=34$ days (since Jan. 1, 2002).

As can be seen from Table 1, the centre of the echo migrates from the central star. The migration keeps more or less the same direction and the distance between the echo centre and the star increases roughly linearly with time since the zero age. This is what is expected from the plane geometry of the dust distribution (see Eq. (7)). It cannot be reconciled with a spherically symmetric distribution. Thus the shape of the outer edge of dust in front of the star can be approximated by a plane inclined to the line of sight rather then a sphere centred on the star. 
Table 2. Results of fitting a circle centred on the star to the northwest quadrant $(x>0, y>0)$ of the outer edge of the light echo of V838 Mon. Time of observations, $t_{\mathrm{obs}}$, is in days since January 1, 2002. Results are in arcsec.

\begin{tabular}{cc}
\hline \hline$t_{\text {obs }}($ days $)$ & $\theta$ \\
\hline 120.0 & $18.46 \pm 0.32$ \\
140.0 & $20.59 \pm 0.19$ \\
245.0 & $28.83 \pm 0.36$ \\
301.0 & $32.83 \pm 0.44$ \\
351.0 & $36.02 \pm 0.45$ \\
\hline
\end{tabular}

A linear fit to the observed evolution of the distance of the echo centre from the star as given in Table 1 results in a relation

$\theta_{\mathrm{c}}=\left(0 .{ }^{\prime} 0101 \pm 0.0026\right)\left(\frac{t}{1 \text { day }}\right)$

where $t=t_{\mathrm{obs}}-t_{0}$ is the time since the zero age of the echo. This with Eq. (7) allows to derive the inclination of the dust surface to the line of sight, i.e.

$\tan \alpha=(0.058 \pm 0.015)\left(\frac{d}{1 \mathrm{kpc}}\right)$.

Unfortunately the distance cannot be determined with a satisfactory accuracy (see below). Adopting $d=5 \mathrm{kpc}$ one obtaines $\alpha \simeq 16^{\circ}$ whereas for $d=10 \mathrm{kpc} \mathrm{Eq}$. (20) gives $\alpha \simeq 30^{\circ}$. Note that the azimuthal angle of the normal to the dust surface is opposite (i.e. $+180^{\circ}$ ) to that of the echo centre.

A least square fit of Eq. (17) to the observed values of $\theta$ in Table 1 gives the best fit for $d \simeq 6.25 \mathrm{kpc}$ and $z_{0} \simeq 2.2 \mathrm{pc}$. However the $\chi^{2}$ minimum is rather shallow and extended along a $z_{0} \sim d^{2}$ relation. From a $90 \%$ confidence level on the $\left(d, z_{0}\right)$ plane we can only state that the lower limit to the distance is $\sim 2.9 \mathrm{kpc}$.

Clearly we are well before the phase when the distance can be unambigiously determined, i.e. when $\left(2 z_{0}\right) /(c t) \ll 1$. The large uncertainty in our distance estimate is also due to uncertainties in the observed values of the echo radius, as given in Table 1. They are simply due to the fact that the echo edge is not ideally reproduced by a circle. Evidently the outer edge of the dust distribution in front of V838 Mon is not a perfect plane.

We have found, however, that in the north-west quadrant, i.e. for $x>0$ and $y>0$, the echo edge is quite well defined and its shape can be well reproduced by a circle centred on the star. This, following the discussion in Sect. 2, implies that the dust edge is here either perpendicular to the line of sight or forms a partial sphere centred on the star. As we shall see below in this paper the former possibility is more consistent with the data.

The results of a least square fit of a circle centred on the star to the observed echo rim in the north-west quadrant are given in Table 2. As can be seen from Table 2 the fit is significantly better than that in Table 1 .

A least square fit of Eq. (17) to the data in Table 2 gives the best fit for $d \simeq 9.0 \mathrm{kpc}$ and $z_{0} \simeq 4.5 \mathrm{pc}$. Although the $90 \%$ confidence region is now more confined than in the previous case, the uncertainty in the distance is still large. All what can be said is that the distance to V838 Mon is greater than $\sim 4.8 \mathrm{kpc}$.
An attempt to fit a sphere, i.e. Eq. (18), to the data in Table 2 has given less consistent results. The $\chi^{2}$ test was unable to find a minimum. It gives acceptable fits starting from $d \simeq 7 \mathrm{kpc}$ but finds better and better fits for increasing distances, well beyond reasonable limits. Clearly the test "prefers" the situation in which the sphere section illuminated by the echo is closer and closer to a plane. This suggests that the constant $z$ model better approximates the outer edge of dust in the north-west quadrant of the V838 Mon echo than the spherical model.

\section{Structure of the dust region near V838 Mon}

The structure of the light echo observed in V838 Mon is rather complex especially in the last three images. This obviously reflects the complex distribution of the circumstellar dust. We have attempted to study this distribution from an analysis of the available images. Of course only dust illuminated by the light echo can be studied which limits the analysis mainly to the regions lying in front of V838 Mon. The results referring to the global dust distribution are presented in Sect. 4.1; Sect. 4.2 analyses the structure of the dust-free region arround the central object.

\subsection{Dust distribution}

In the available echo images, apart from the outer edge, one can also distinguish emission edges inside the echo. These edges are usually either blue or red in colour. From the light curve of V838 Mon it is clear that the object was bluest at the beginning of the outburst (beginning of February 2002) while during the fading (mid-April 2002) it was extremely red (Munari et al. 2002a; Bond et al. 2003). Suppose that the paraboloids $c t=2$ and $c t=1$ in Fig. 1 correspond to the blue beginning and the red end of the light flash, respectively. Suppose also that, say, between $z=5$ and $z=10$ there is a dust layer. Then the observer would see an echo ring whose outer edge would be blue and would correspond to the intersection of $z=10$ with $c t=2$, while the inner edge would be red and would correspond to the intersection of $z=5$ with $c t=1$. Thus measurements of the positions of the blue and red edges in the light echo of V838 Mon can be used to estimate positions of the boundaries of dust layers producing bright regions in the echo.

We have measured, apart from the outer edge discussed in Sect. 3, positions of blue and red edges inside the echo images. This concerns primarily the images taken on Sep. 2, Oct. 28 and Dec. 17 showing several well defined details in the echo structure. In the image from May 20 only the inner red edge has been measured (apart from the outer blue edge). On Apr. 30 only a B image was taken so no (inner) red edge can be identified. Following the above considerations we assume that the blue edges are produced by the beginning of the outburst so, as discussed in Sect. 3, they correspond to the light paraboloid with $t_{0}=34$ days. From the I curve in Bond et al. (2003) it can be found that near Apr. 17 V838 Mon declined by factor 2 from its last peak. Therefore we assume that the red edges in the light echo correspond to the paraboloid with $t_{0}=107$ days.

Knowing $x$ and $y$ from measurements of an edge in the echo observed at a given time, $t$, one can calculate the $z$ coordinate 

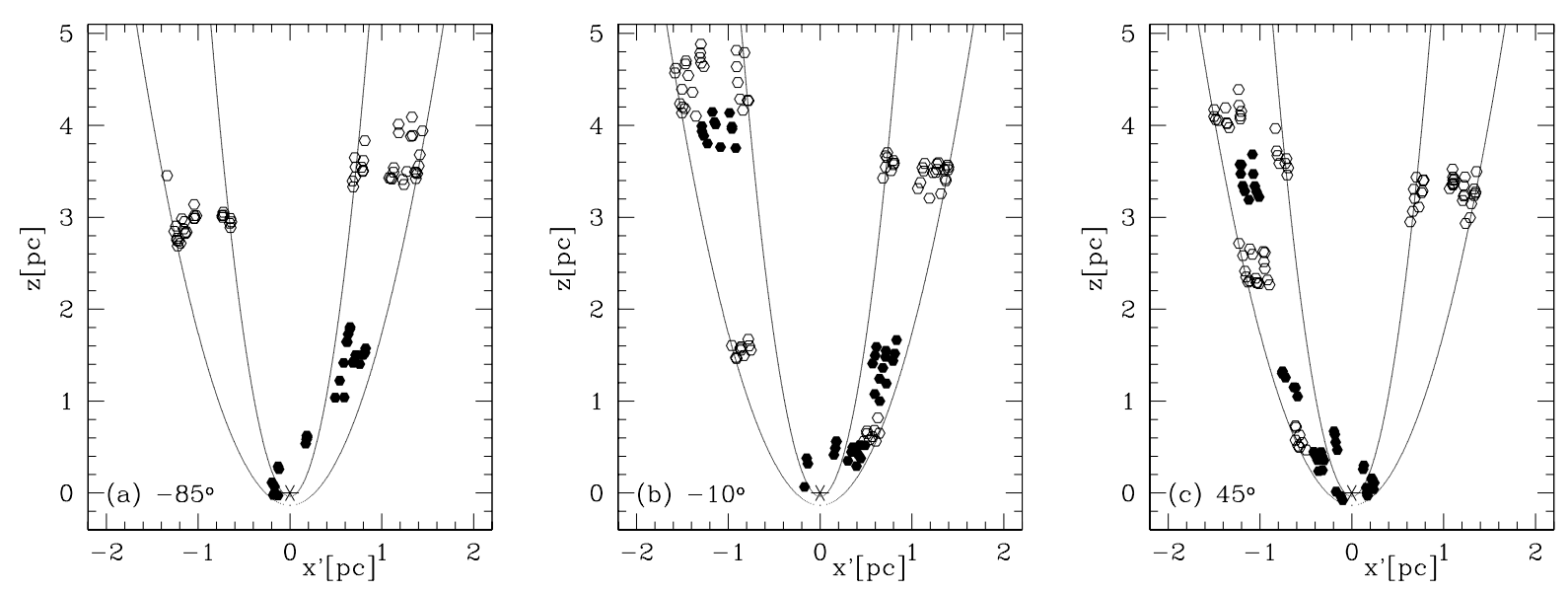

Fig. 4. Structure of the dust distribution illuminated by the lihgt echo of V838 Mon. In all the images the ordinate is the $z$ axis. The abscissa, $x^{\prime}$, is inclined to the $x$ (east-west) axis at an angle, $\beta$, (clockwise in respect to the $x$ axis) given in the bottom-left corner of each image. Only the points having the position angle within $\beta \pm 15^{\circ}$ are shown in each image. Open and full symbols correspond to upper and lower (in the $z$ coordinate) boundaries of the dusty regions, respectively. Thin curves show the echo paraboloids (with $t_{0}=34$ days) on Apr. 30 and Dec. 17 . The position of the central star is shown by an asterisk.

of the dust edge from Eq. (3). Obviously we measure only the angular values of $x$ and $y$ so in order to have their absolute values we have to assume the distance. Following the analysis done later on in Sect. 4.2 we assume that V838 Mon is at a distance of $8 \mathrm{kpc}$.

The results on the dust structure are presented in Fig. 4. It shows three cuts across the three dimensional distribution of the dust boundaries inferred from the measurements of the blue (open points) and red (full points) edges in the light echo. The plane of each panel of Fig. 4 goes through the $z$ axis and is inclined to the $x$ (east-west) axis at an angle, $\beta$, (counted clockwise from the $x$ axis) given in the bottom-left corner of each part of the figure. Only the points having an azimuthal angle within $\beta \pm 15^{\circ}$ are shown. The scale of the axes is in parsecs. Thin curves show the echo blue paraboloids (with $t_{0}=$ 34 days) on Apr. 30 and Dec. 17. Thus, in principle, we can study only the dust distribution between these two paraboloids. The exception is the inner red edge on May 20 whose (red) paraboloid lies within the (blue) paraboloid on Apr. 30. Dust is present above the full and below the open symbols (it is absent above the open and below the full points).

The cuts shown in Fig. 4 have been chosen so as to show the global distribution of dust. They are more or less equally spaced in the azimuthal angle $\beta$ but they also avoid too detailed structures which would make the discussion too complicated and less clear. We recommend consulting the original echo images (e.g. at http://hubblesite.org/ newscenter/archive/2003/10/) for an easier understanding of the discussion below.

Figure $4 \mathrm{a}$ shows the cut done close to the south-north direction. It goes more or less across the major axis of the central hole in the echo images. In the southern part $\left(x^{\prime}<0\right.$ in Fig. 4a) dust extends from the inner boundary which lies very close to $(\sim 0.15 \mathrm{pc}$ from) the central object up to $\sim 3 \mathrm{pc}$ in $z$. In the northern part $\left(x^{\prime}>0\right)$ the inner dust boundary is much futher away, i.e $\sim 1.5 \mathrm{pc}$, from the central object. Then dust goes up to $\sim 3.5 \mathrm{pc}$.
The section in Fig. $4 \mathrm{~b}$ goes close to the east-west direction. In the eastern part $\left(x^{\prime}<0\right)$ dust starts rather close to the central star (similarily as for $x^{\prime}<0$ in Fig. 4a) but extends only up to $\sim 1.5 \mathrm{pc}$. Then above there is an empty region. Only at $\sim 4.5 \mathrm{pc}$ there is a thin dust layer. On the western side of the section $\left(x^{\prime}>0\right)$ there is a thin dust layer at $\sim 0.7 \mathrm{pc}$. Then the main dust layer extends between $\sim 1.5$ and $\sim 3.5 \mathrm{pc}$.

The last section presented in Fig. $4 \mathrm{c}$ has been made along the north-east - south-west direction. In the north-eastern part $\left(x^{\prime}<0\right)$ the dust structure is rather complex. There are two thin dust layers seen at $\sim 0.7 \mathrm{pc}$ and $\sim 3.7 \mathrm{pc}$. Between them there is a much thicker dust region which extends from $\sim 1.0 \mathrm{pc}$ to $\sim 2.5$ pc. Note however that this region has its own complex structure as can be best inferred from the echo image taken on Dec. 17. In the south-western part an extended dust region begins at $\sim 0.15 \mathrm{pc}$ from the star and goes up to $\sim 3.3 \mathrm{pc}$.

The general picture that emerges from our analysis is as follows. Near the central object there is a dust-free region (which is separately discussed in the following subsection). Above it there is the main dust region whose outer boundary to the north, west and south lies at $\sim 3.0-3.5 \mathrm{pc}$. In all eastern directions the boundary is closer to the star and near the east axis it is as close as $\sim 1.5 \mathrm{pc}$. Finally, in the eastern directions there is a thin dust layer at a distance of $\sim 4 \mathrm{pc}$.

Note that the obtained positions of the dust regions depend on the assumed distance to V838 Mon. The most sensitive is the $z$ coordinate which depends on the square of the distance. Fortunatelly, the relative picture of the dust distribution presented in Fig. 4 would be little affected if other values of the distance were adopted.

In Sect. 3 we have interpreted the outer edge of the observed echo in the north-west quadrant as produced by a flat surface perpendicular to the line of sight. From the results in the present section it results that it was a reasonable assumption. The $z$ values of the outer rim points from this quadrant (i.e. having $x>0, y>0$ ) are scattered by less than $3 \%$ (standard deviation) around its mean value of $3.50 \mathrm{pc}$. 


\subsection{Central dust-free region}

As mentioned above our analysis of the echo images of V838 Mon leads to the conclusion that there is a quite well defined dust-free region around the central object. This statement obviously results from the fact that all the echo images analysed in the present study show a clear central hole. All the images have been taken after the outburst of V838 Mon. The light echo has then been well separated from the line of sight in front of the star. Thus, as can be seen from Fig. 1 or Fig. 4, the central parts of the echo tells us about the dust distribution behind the star and in the directions from the star more or less perpendicular to the line of sight.

The principal characteristic of the central hole in the light echo observed around V838 Mon is that it expands strongly asymmetrically. This immediately implies that the dust-free region around the central object is strongly asymmetric. The hole expands most rapidly in the north and north-west directions. In the east, south-east and south directions it expands very little or even not at all.

Another important characteristic is that the inner echo edge is well defined, in the sense that it is usually marked by bright emission regions or bright rims. In particular, clear rim-like structures are seen in the east, south-east and south directions. They are most pronounced on Sep. 2 but can be easily identified more or less in the same distance from the central star in all the images.

On Oct. 28 an emission appears in the south-east quadrant between the rim and the central star. On Dec. 17 this central emission becomes extended to the north-east. Evidently on Oct. 28 we started seeing dust behind the central object.

The bright rim, relatively stable and seen for $\sim 8$ months implies that to the south, south-east and east of the central star there is a sort of a dust layer more or less parallel to the line of sight. We suggest that this is a compressed edge of a cavity more or less centred on the star, whose illuminated parts are roughly tangent to the line of sight. This interpretation is supported by the appearance of the emission from behind the star on Oct. 28 and its futher growth on Dec. 17, as discussed above.

The dense edge of the cavity with a geometry as suggested above offers a possibility to estimate the distance to V838 Mon. At a certain time moment the light echo would illuminate parts of the edge which lie at $z=0$. The distance of these parts from the central star would be equal to $c t$ at this moment (cf. Eq. (6)). The main problem is to identify the right moment. Bond et al. (2003) have attempted to explore this possibility following considerations of Sparks (1994). They have detected that on Sep. 2 the light from the discussed rim was strongly polarized indicating that it was scattered at an angle close to $90^{\circ}$. From this they conclude that the distance is at least $6 \mathrm{kpc}$, suggesting that the $90^{\circ}$ scattering in the rim had not yet occured. Our measurements on the images from Sep. 2, Oct. 28 and Dec. 17 give that the inner bright rim in the south-east quadrant is practically at the same distance of $4.35^{\prime \prime} \pm 0.55^{\prime \prime}$ from the central star. (Note that the uncertainity mainly results from the fact that the rim is not perfectly circular.) On May 20 the same rim was at $4.11^{\prime \prime} \pm 0.15^{\prime \prime}$. Apparently on May 20 the rim resulted from illumination of the regions in front of the star, i.e. at $z>0$. On Oct. 28 and particularly on Dec. 17 the light echo was already partly illuminating dust behind the star, i.e. at $z<0$. Thus it seems reasonable to adopt that the maximum illumination of the rim at $z=0$ occured between Sep. 2 and Oct. 28. Taking that this has happened at $t_{\mathrm{obs}}=270 \pm 30$ days and that the zero age of the echo is $t_{0}=70 \pm 30$ days (middle of the main outburst and half duration of the outburst) one obtains the distance to V838 Mon of $8.0 \pm 2.0 \mathrm{kpc}$.

In order to study more quantitatively the structure of the central dust-free region we have remeasured the positions of the inner echo edge on the images taken on May 20, Sep. 2, Oct. 28 and Dec. 17. Contrary to the previous measurements reported above, which referred to regions where the emission became almost invisible, we have measured the positions where, going from the central star, the echo emission becomes significant. In particular when the edge is marked by a bright rim the position of the maximum emission in the rim has been determined. In addition, on the images from Oct. 28 and Dec. 17, we have also measured the positions of the emission appearing in the south-east quadrant between the inner rim and the star.

We do not know when, i.e. in which part of the outburst, the light illuminating the measured regions was emitted. Therefore when calculating the $z$ coordinates of these regions from Eq. (3) we have assumed $t_{0}=70$ days, which is the time of the middle of the main outburst. Following the above estimate, the $8 \mathrm{kpc}$ distance to V838 Mon has been assumed.

The resulting structure of the inner edge of the dusty region surrounding V838 Mon is shown in Fig. 5. The axes and the azimuthal angle of the abscissa, $\beta$, given in the bottom-left corner of each section, are defined in the same way as in Fig. 4. Full symbols show the positions of the inner edge of dust. Cometlike tails of the symbols have been drawn to indicate directions in which dust extends. Inside, i.e. between the full points and the central star, there is no (observable) dust.

The plane of Fig. $5 \mathrm{a}$ is $15^{\circ}$ inclined to the west from the south-north axis in the echo images. The dust-free region is here most asymmetric. For $x^{\prime}<0$ and $z \lesssim 0$ the dust edge is as close as $0.10-0.15 \mathrm{pc}$ from the star. In front of the $\operatorname{star}(z>$ 0 ) the dust edge increases its distance from the star with the increasing $x^{\prime}$ up to $\sim 1.5 \mathrm{pc}$. We do not have information about the dust distribution beyond the outer paraboloid in Fig. 5 but the dust-free region certainly extends beyond this paraboloid in Fig. 5a for $x^{\prime}>0$ and $z \lesssim 1.3 \mathrm{pc}$.

The distribution of the points in Fig. 5c, whose plane is perpendicular to that of Fig. 5a, is fairly symmetric in relation to the $x^{\prime}=0$ line. The planes fo Figs. $5 \mathrm{~b}$ and $\mathrm{d}$ are inclined $45^{\circ}$ and $-45^{\circ}$ to the plane of Fig. 5a. The distribution of the points in Fig. $5 \mathrm{~b}$ is almost a mirror image of that in Fig. 5b. Therefore it can be concluded that the dust-free region shows a noticable symmetry with respect to the plane of Fig. 5a. Note, however, that the symmetry is not perfect. In Fig. $5 d$ we see almost a complete shape of the dust edge around the central object. In Fig. 5b, however, the dust-free region extends beyond the outer paraboloid for $x^{\prime}>0$ and $z \lessgtr 0.4 \mathrm{pc}$.

The (compressed) dust edge producing the bright inner echo rim discussed above (and which have been used to estimate the distance to V838 Mon) is clearly seen as a 

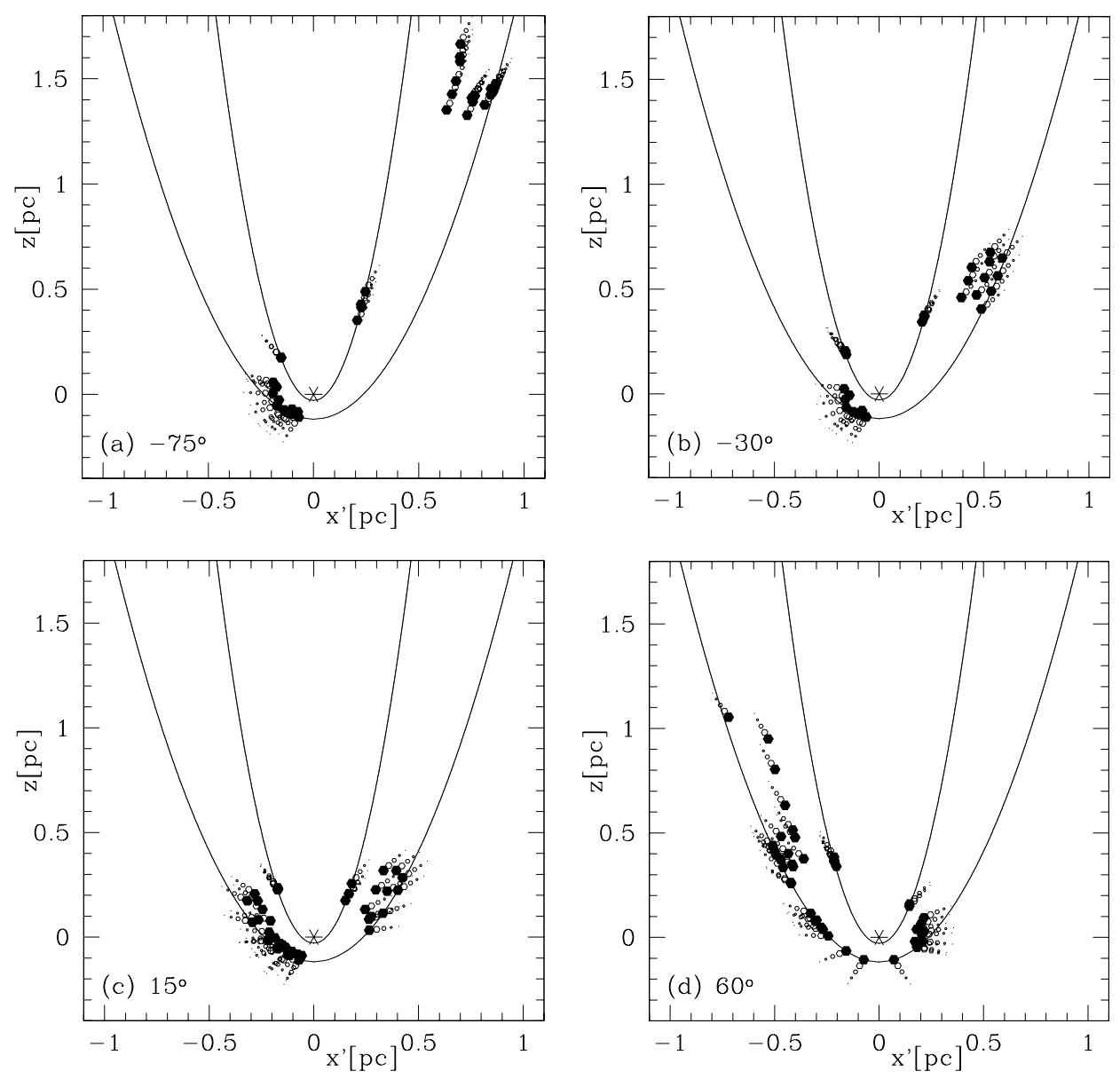

Fig. 5. Structure of the dust-free region near V838 Mon. The axes and the definition of the azimuthal angle, $\beta$, given in the bottom-left corner of each part, are the same as in Fig. 4. Full symbols show the positions of the inner edge of dust (within $\beta \pm 15^{\circ}$ ). Comet-like tails emerging radially from the symbols indicate directions in which dust extends. Thin curves show the echo paraboloids corresponding to $t_{0}=70$ days on May 20 (inner) and Dec. 17 (outer).

concentration of points near the central star at $x^{\prime}<0$ in Figs. 5a and $\mathrm{b}$ and at $x^{\prime}>0$ in Fig. 5d.

\section{Discussion and conclusions}

For all astrophysical objects it is important to know their distances. In the case of V838 Mon this is particularly important. The outburst of V838 Mon does not fit any known class of outbursting stars so the determination of its luminosity is crucial for discussing possible mechanisms of the event. The early estimates of $0.6-0.8 \mathrm{kpc}$ based on a naive interpretation of the light echo expansion (Munari et al. 2002a; Kimeswenger et al. 2002) led to the maximum luminosity of $\sim 10^{4} L_{\odot}$ which is typical for nova or nova-like outbursts. Our analysis presented in Sect. 3, similar to that of Bond et al. (2003), clearly shows that the distance and thus also the luminosity are much larger. The observed expansion of the outer echo edge led us to conclude that the distance to V838 Mon is $\gtrsim 5 \mathrm{kpc}$. From an analysis of the inner rim of the echo done in Sect. 4.2 we have estimated the distance to be $8.0 \pm 2.0 \mathrm{kpc}$. This distance estimate is in agreement with that of Bond et al. (2003), as well as with that of Munari et al. (2002b). Even our lower limit of $\sim 5 \mathrm{kpc}$ makes the maximum luminosity $\gtrsim 10^{6} L_{\odot}$ which places the outburst of V838 Mon among the most luminous events in our Galaxy.

As it has been shown in Sect. 4 the evolution of the light echo can be used as a very useful tool for studying the dust distribution near the light source. Although the absolute characteristics of the obtained distribution depend on the distance, which is uncertain in the case of V838 Mon, the relative structure is fairly insensitive to this parameter. What we have done it is a rather simple analysis. A much more detailed study could be done on calibrated images of the echo which were not available to the author. Certainly further observations would be very valuable. When the light paraboloid becomes larger and larger, newer and newer regions of dust are illuminated and more and more complete images of the dust distribution in the vicinity of the star can be obtained. As it can be seen from Fig. 4, only a small part of the volume around V838 Mon could be studied so far. But even from this interesting conclusions can be drawn.

We have found no signs of spherical symmetry in the dust distribution which would have been expected if the observed dust had resulted from mass loss activities of V838 Mon in the past. Thus the results of our study contradict the assumption made in Bond et al. (2003) that the V838 Mon light echo has been produced by "a series of nested spherical dust shells 
centered on the star". In fact, even a simple qualitative analysis of the echo images leads to the conclusion that the assumption of Bond et al. is doubtful. Numerous ring-like structures seen on the images of Apr. 30 and May 20 might be considered as justifying the assumption of spherical symmetry. However, one has to keep in mind that the echo image is determined by two, to some extent competing, factors: dust distribution and shape of the light echo paraboloid. The latter is symmetric relatively to the line of sight so in the echo image it will always tend to give features symmetric relative to the central star. At early epochs when the opening angle of the echo paraboloid is small the shape of the echo is dominant in structuring the echo image. Thus it is not surprising that the images of Apr. 30 and May 20 show so many symmetric or ring-like features. But it also implies that they say little about the dust distribution. At later epochs, when the echo paraboloid becomes more and more open, details in the dust distribution become more and more important in producing the resulting echo image. Thus the fact that numerous ring-like structures seen on Apr. 30 and May 20 become less and less evident in later images and that the light echo develops more and more asymmetric features implies that the dust distribution is asymmetric.

The outer edge of the dust distribution in front of V838 Mon, studied in Sect. 3, if tentatively fitted by a simple geometry gives a plane inclined to the line of sight at $\sim 25^{\circ}$ (for $d=8 \mathrm{kpc}$ ). The detailed study of the dust distribution done in Sect. 4.1 shows that the main dust region in the western part has the outer boundary relatively flat and almost perpendicular to the line of sight. Near the south-north line it bends toward the central star or rather splits into a thin outer layer and a thicker zone closer to the central star.

Near the central object there is a dust-free region. Our detailed analysis of the central hole in the echo images done in Sect. 4.2 has shown that this dust-free region is strongly asymmetric. Its boundary is quite close to the star in the southern directions but in the northern directions it is at least 10 times further away. Holes near central stars of e.g. planetary nebulae or HII regions, are often observed. They are produced by fast winds from the stars which sweep out the nebular matter. V838 Mon has a B3V companion (Munari et al. 2002b). It is also plausible that V838 Mon itself was also of similar spectral type before the outburst (Tylenda, in preparation). According to the standard calibration (Drilling \& Landolt 2000) B3V stars have $\log L / L_{\odot} \simeq 3.5$ which, using the mean relation of Howarth \& Prinja (1989), turns into a mass loss rate of $\sim 3 \times 10^{-10} M_{\odot} / \mathrm{yr}$. Thus, it is quite probable that the fast wind from the V838 Mon system has created the central hole in the dusty medium. The fact that the hole is strongly asymmetric would imply that V838 Mon is moving relative to the dusty medium. The bright inner echo rim to the east, south-east and south discussed in Sect. 4.2 and the shape of the inner edge of dust in these directions displayed in Fig. 5 fit almost perfectly the idea that these are regions compressed by the wind ahead of the moving star. The structure of the dust-free region shown in Fig. 5 suggests that the direction of the V838 Mon movement relative to the dusty medium lies close to the plane of Fig. 5a and that the star moves in the south-south-east direction. The angle between this direction and the line of sight is uncertain as we cannot see the other side of the dust-free region in Fig. 5a. It seems, however, to be almost certainly lower than $150^{\circ}$ and probably greater than $90^{\circ}$.

The above conclusion that V838 Mon is moving relative to the dusty medium would be difficult to understand if the medium were produced by mass loss from V838 Mon in the past. It is however quite natural if the dust is of interstellar origin. We therefore conclude that dust illuminated by the light echo of V838 Mon is most probably of interstellar origin. This is also supported by the general lack of spherical symmetries in the dust distribution, as discussed above. Thus the presence of circumstellar dust seen in the light echo is not evidence that V838 Mon had several outbursts in the recent past as claimed by Bond et al. (2003). Consequently their criticism of the merger scenario proposed by Soker \& Tylenda (2003) is not relevant. Note, however, that in view of the distance to V838 Mon being much larger than $1 \mathrm{kpc}$, the scenario of Soker $\&$ Tylenda should be revised (which will be done in a separate paper).

Finally, as it is evident from the analysis and discussion made in the present paper, future observations of the V838 Mon light echo would be of particular importance and interest.

Acknowledgements. The author is very grateful to Noam Soker for his comments on the initial version of the paper and to the referee (F. Patat) whose thorough remarks were very helpful while improving the final version of the paper.

\section{References}

Bond, H. E., Henden, A., Levay, Z. G., et al. 2003, Nature, 422, 405 Brown, N. J. 2002, IAU Circ., 7785

Cappellaro, E., Patat, F., Mazzali, P. A., et al. 2001, ApJ, 549, L215

Chevalier, R. A. 1986, ApJ, 308, 225

Couderc, P. 1939, Ann. Astrophys. 2, 271

Crotts, A. P. S. 1988, ApJ, 333, L51

Drilling, J. S., \& Landoldt, A. U. 2000, in Allen's Astrophysical Quantities, the 4th edition, ed. A. N. Cox (New York: SpringerVerlag), 381

Henden, A., Munari, U., \& Schwartz, M. B. 2002, IAU Circ., 7859

Howarth, I. D., \& Prinja, R. K. 1989, ApJS, 69, 527

Kimeswenger, S., Lederle, C., Schmeja, S., \& Armsdorfer, B. 2002, MNRAS, 336, L43

Munari, U., Henden, A., Kiyota, S., et al. 2002a, A\&A, 389, L51

Munari, U., Desidera, S., \& Henden, A. 2002b, IAU Circ., 8005

Retter, A., \& Marom, A. 2003, MNRAS, 345, L25

Schmidt, B. P., Kirshner, R. P., Leibundgut, B., et al. 1994, ApJ, 434, L19

Soker, N., \& Tylenda, R. 2003, ApJ, 582, L105

Sparks, W. 1994, ApJ, 433, 19

Sugerman, B. E. K., \& Crotts, A. P. S. 2002, ApJ, 581, L97

Xu, J., Crotts, A. P. S., \& Kunkel, W. E. 1995, ApJ, 451, 806 